\title{
Acoustophoresis in suspensions with local- and time- discrete sound fields based on the time reversal technique
}

\author{
Philipp Hörnlein ${ }^{1}$, Sebastian Wöckel ${ }^{1}$, Hendrik Arndt ${ }^{1}$, Jörg Auge ${ }^{2}$ \\ ${ }^{1}$ Institute for Automation and Communication (ifak) e. V., 39106 Magdeburg, Germany \\ ${ }^{2}$ University of Applied Sciences Magdeburg-Stendal, 39114 Magdeburg, Germany \\ Philipp.Hoernlein@ifak.eu
}

\begin{abstract}
Summary:
This contribution presents a new approach concerning the acoustic 3D-manipulation of particles in a liquid filled volume. Based on simulation studies and a physical model, the realization of acoustophoresis using the acoustic radiation force (ARF) on particles in a liquid-filled vessel is discussed. The discrete local acoustic pressure field, that is required for the acoustophoresis, is generated by a time reversal mirror consisting of 24 piezoceramic transducers.
\end{abstract}

Keywords: acoustophoresis, acoustic tweezers, levitation, particle manipulation, time reversal

\begin{abstract}
Motivation
The manipulation of particles and cells in fluids has numerous applications in medicine and biotechnology. This complies amongst the sorting and separation in sense of size and material, especially the exact positioning of single cells for further investigation or biochemical treatment. In this context the acoustophoresis is a proven method that works contactless, biocompatible and label-free [1]. Existing methods use either volume waves, surface waves or focused fields (acoustic vortexes) [2] to manipulate objects. Focused acoustic fields can be used to achieve a very specific stationary force effect on one or more objects (acoustic tweezers). Such previous rely either on phased array technology [3], passive systems (resonator [4], phase plate [5], diffraction grating [6]) or special transducer geometries [7]. However, these techniques have limitations when focusing is to be performed in a closed room or vessel (cavity). In addition, passive systems and special transducer geometries provide stationary acoustic fields only and do not allow an electronic translational displacement of the objects.
\end{abstract}

\section{Methods}

In order to circumvent the previous limitations, this contribution discusses the use of the ultrasonic time reversal (TR) technique to generate a transient sound field in a liquid-filled vessel. In the variant of the time reversal technique used here, the transfer function or impulse response from the piezoceramic ultrasonic transducers to the desired focal points is determined by means of a suitable measurement setup (calibration) [8]. By time reversal of the impulse responses, the time reversal signal is formed, whose emission at the associated transducer leads to a high-quality focusing on the target point. Almost any sound field geometry can be generated by simultaneous response of several focus points. The advantage is that, especially in small vessels - like bioreactors -, the reflection processes in the cavity contribute significantly to the focusing effect, which is not the case with previous realizations. In addition, the sound field geometry and its position can be varied (moved) arbitrarily within the cavity.

\section{Results}

The feasibility of the transient time-reversal focusing and the generation of specific complex sound field geometries, in the sense of particle traps, were investigated using 3D-simulations with COMSOL Multiphysics. On the basis of these parameter studies it could be shown, that a so-called bottle trap represents an ideal sound field geometry for this purpose in terms of local pressure field, reproducibility and particle force vector. The trap consists of six focus points which are excited simultaneously. In the case of the bottle trap, there are two focus points in each axis of a cartesian coordinate system, which are opposite each other at a defined distance - depending on the physical parameters of the particle and the surrounding medium. The object to be manipulated is located between the focal points. In this way, a three-dimensional force effect based on the ARF is achieved. The investigation shows that such a sound field based on the time reversal technique is basically feasible if the time reversal signals are at least as long as a sound wave needs to pass through the largest dimension of 
the vessel five times. Furthermore, it becomes clear that focal points which are close to each other and have the same phase position merge to one focal point. It is therefore obvious that opposite focal points have an inverse sound pressure amplitude. If opposing focus points have an additional distance of $N 2$, an acoustic field is realized which in principle corresponds to a locally limited standing wave in three dimensions (Fig 1). By high-frequency repetition of the time reversal signals, a quasi-stationary sound field can be generated. As prove of example, investigations with 24 single element transducers (in the range from $250 \mathrm{kHz}$ to 1 $\mathrm{MHz}$ ) show, that force effects in all three dimensions (F0), which allow a levitation and manipulation of particles from various plastics, glass and graphite in the order of $N 10$, are already achievable with low excitation voltages of 20 VPP.

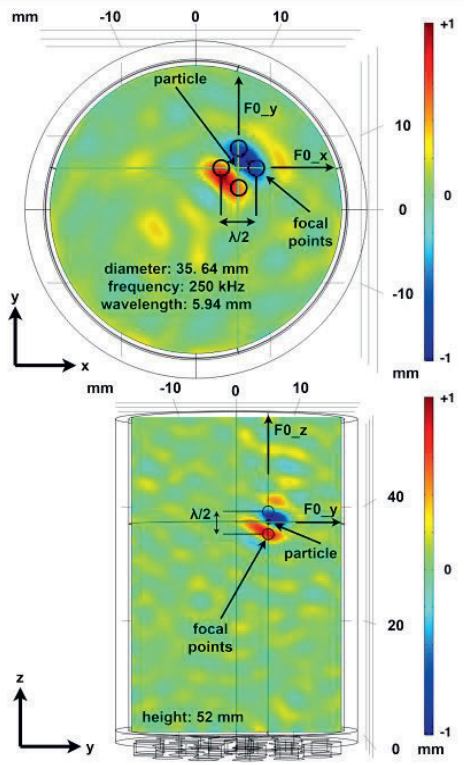

Fig. 1. Simulation-example at $250 \mathrm{kHz}$ : Intensity plot of the acoustic pressure field of the bottle trap (top) in the $X Y$ and (bottom) in the $Y Z$ plane in a cylindrical vessel with piezoceramic transducers on the bottom.

\section{Experimental Setup and Outlook}

Based on the results of the simulations a demonstrator has been realized (Fig. 2). It consists of a cylindrical vessel (diameter $35.64 \mathrm{~mm}$, height $52 \mathrm{~mm}$ ) made of PMMA (wall thickness $1.36 \mathrm{~mm}$ ) with 24 piezoceramic transducers (PIC255) with a diameter of $4.9 \mathrm{~mm}$ each coupled to its bottom side. The transducers operate at a frequency of $1 \mathrm{MHz}$. The vessel is filled with distilled water and the bottom serves as a N/4 matching layer for low-reflection coupling of the transducers to the liquid. In the further course of the work a suitable control system has to be designed and realized. This will probably consist of an FPGA and a multi-channel amplifier.

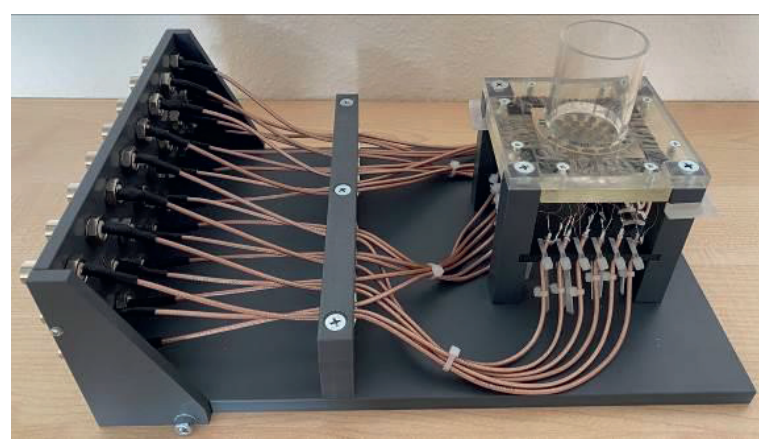

Fig. 2. TR demonstrator for $1 \mathrm{MHz}$ with 24 single transducers and a cylindrical cavity for liquid fillings.

\section{Conclusion}

Within the work it could be shown by means of simulation studies, that on the basis of the time reversal technique an acoustic field can be realized which enables the transient acoustophoresis in liquid filled vessels. The so-called bottle trap, which can be moved translatorily in the vessel, was determined as the ideal sound field geometry. In addition, a suitable structure in the form of a demonstrator was shown, for which a suitable control system needs to be developed afterwards.

\section{References}

[1] A. Lenshof et al., "Acoustophoresis," in Encyclopedia of Nanotechnology, B. Bhushan, Ed. Dordrecht: Springer Netherlands, 2014, pp. 1-6

[2] A. Ozcelik et al., "Acoustic tweezers for the life sciences," Nature Methods, vol. 15, no. 12, pp. 1021-1028, 2018, doi: 10.1038/s41592-0180222-9

[3] A. Marzo et al., "Holographic acoustic elements for manipulation of levitated objects," Nature Communications, vol. 6, no. May, pp. 1-7, 2015, doi: $10.1038 /$ ncomms 9661

[4] X. Jiang et al., "Convert Acoustic Resonances to Orbital Angular Momentum," Physical Review Letters, vol. 117, no. 3, 2016, doi: 10.1103/PhysRevLett.117.034301

[5] M. E. Terzi et al., "Generation of a vortex ultrasonic beam with a phase plate with an angular dependence of the thickness," Moscow University Physics Bulletin, vol. 72, no. 1, pp. 61-67, 2017, doi: $10.3103 /$ S0027134916050180

[6] N. Jiménez et al., "Formation of high-order acoustic Bessel beams by spiral diffraction gratings," Physical Review E, vol. 94, no. 5, pp. 1-18, 2016, doi: 10.1103/PhysRevE.94.053004

[7] M. Baudoin et al., "Folding a focalized acoustical vortex on a flat holographic transducer: Miniaturized selective acoustical tweezers," Science Advances, vol. 5, no. 4, pp. 1-7, 2019, doi: 10.1126/sciadv.aav1967

[8] Y. D. Sinelnikov et al., "Time reversal in ultrasound focusing transmitters and receivers," Acoustical Physics, vol. 56, no. 2, pp. 183-193, 2010, doi: 10.1134/S1063771010020107 